\title{
Numerical study of the reconnection process between magnetic cloud and heliospheric current sheet
}

\author{
Man Zhang ${ }^{1}$, Yu Fen Zhou ${ }^{1}$, Xue Shang Feng ${ }^{1}, \mathrm{Bo} \mathrm{Li}^{2}$, and Ming Xiong ${ }^{1}$ \\ 1 SIGMA Weather Group, State Key Laboratory for Space Weather, National Space Science Center, Chinese Academy of Sciences, \\ 100190 Beijing, PR China \\ e-mail: mzhang@spaceweather.ac.cn; yfzhou@spaceweather.ac.cn; fengx@spaceweather.ac.cn \\ 2 Shandong Provincial Key Laboratory of Optical Astronomy and Solar-Terrestrial Environment, School of Space Science and \\ Physics, Shandong University at Weihai, 264209 Weihai, PR China
}

Received 5 March 2018 / Accepted 20 August 2018

\begin{abstract}
In this paper, we have used a three-dimensional numerical magnetohydrodynamics model to study the reconnection process between magnetic cloud and heliospheric current sheet. Within a steady-state heliospheric model that gives a reasonable large-scale structure of the solar wind near solar minimum, we injected a spherical plasmoid to mimic a magnetic cloud. When the magnetic cloud moves to the heliospheric current sheet, the dynamic process causes the current sheet to become gradually thinner and the magnetic reconnection begin. The numerical simulation can reproduce the basic characteristics of the magnetic reconnection, such as the correlated/anticorrelated signatures in $\boldsymbol{V}$ and $\boldsymbol{B}$ passing a reconnection exhaust. Depending on the initial magnetic helicity of the cloud, magnetic reconnection occurs at points along the boundary of the two systems where antiparallel field lines are forced together. We find the magnetic filed and velocity in the MC have a effect on the reconnection rate, and the magnitude of velocity can also effect the beginning time of reconnection. These results are helpful in understanding and identifying the dynamic process occurring between the magnetic cloud and the heliospheric current sheet.
\end{abstract}

Key words. Sun: coronal mass ejections (CMEs) - Sun: heliosphere

\section{Introduction}

Magnetic reconnection is a fundamental plasma process in which magnetic field changes its topology and magnetic field energy is converted into kinetic and thermal energies (Odstrcil \& Karlicky 1997; Wei et al. 2005). It is widely accepted that magnetic reconnection is significant in the universe, including solar eruptions, geomagnetic substorms and tokamak disruptions (Ugai 2001; Zhang et al. 2011). Reconnection leads to the quickly release of plasma energy stored in the magnetic field, the formation of current sheet and magnetic cloud (MC) in solar corona. It can also leads to the formation of "open" interplanetary magnetospheric field structures and the magnetic flux transfer events at the dayside magnetopause, which plays an important role in the Earth's space weather system (Wei et al. 1997).

In the years since reconnection was first discovered, there have been suggestions that the process might also occur in interplanetary space, for example at the heliospheric current sheet (HCS), which separates oppositely directed magnetic fields originating from the Sun, at the leading edges of interplanetary coronal mass ejections (ICMEs), or at current sheets formed by solar wind turbulence (Lavraud et al. 2009). Recently, Gosling et al. (2005, 2006a,b, 2007), Gosling (2008, 2012), Gosling \& Phan (2013) identified the clear and unambiguous signature of local, quasi-stationary magnetic reconnection in the solar wind far from the Sun in the form of Petschek-type exhausts characterized by bifurcated current sheets, in other words, exhausts of jetting plasma bounded by Alfvén or slow mode waves. These current sheets form a pair of rotational discontinuities bounded on one side by correlated changes in the components of the magnetic field and flow velocity and on the other side by anticorrelated changes in the components. Based on the direct magnetic reconnection evidence, it has been demonstrated that reconnection is particularly frequent in low $\beta$ plasma, such as MCs.

Magnetic reconnection may occur in particular at the boundary of a MC and erodes away part of the magnetic flux contained in the MC, which would affect the impact of a geomagnetic storm as it may remove a substantial portion of the magnetic flux that is oriented southward (Lavraud et al. 2011, 2014; Ruffenach et al. 2014). Ruffenach et al. (2012) use combined, multipoint observations of the same MC flux rope, and quantitatively analyze the MC's reconnection with the solar wind around it, find that $44 \%$ and $49 \%$ of the initial azimuthal magnetic flux are eroded according to estimation at ACE and STEREO A respectively. Lavraud et al. (2014) using a southnorth magnetic polarity MC observed on 18-20 October 1995 and investigate the effectiveness of the geo-effectiveness of MCs with erosion, the results suggest that the strength of the observed ensuing storm was $\sim 30 \%$ lower than if no erosion had occurred. Ruffenach et al. (2014) analyzed 263 MCs observed by Wind, STEREO-A and STEREO-B during the period 1995-2012, they find that MCs have an average erosion of about $40 \%$ of the total azimuthal magnetic flux and the occurrence of erosion at the front or at rear is in a similar proportions.

The observations can only give us a quantitatively analyze of the magnetic reconnection between MC and the solar wind. Numerical simulations were also invoked to study the magnetic reconnection processes. Schmidt \& Cargill (2003) presents a numerical study of magnetic reconnection processes between 
MC and the solar wind magnetic field with a 2.5-dimensional (2.5D) flux-corrected transport algorithm. The solar wind background is a Parker solution. The pressure and density are related by an adiabatic law and the magnetic field is a distorted radial magnetic field (Schmidt 2000). They find that reconnection occurs either symmetrically along both sides of the interface or not at all when a cloud moves in a current sheet, depending on the relative orientation of the cloud and solar wind fields. Wei et al. (2005) shows the numerical study of the magnetic reconnection driven by a plasma blob impacting the HCS, under the framework of the 2D compressible magnetohydrodynamics (MHD). The initial equilibrium state of current sheet is considered to be one-dimensional form. The results show a stable magnetic reconnection structure can be formed in a time scale of hour order, and there appear some basic properties such as the multiple X-line reconnections, vortex structures, filament current systems, splitting and collapse of the high-density plasma blob. Taubenschuss et al. (2010) investigate the propagation of MCs through the inner heliosphere using 2.5D ideal MHD simulations. Interplanetary magnetic fields are oriented in opposite directions across the equator to form a HCS at the equatorial plane. The authors assume that there is no tilt between the Sun's magnetic and rotational axes. There is an enhanced thermal pressure and decreased magnetic field strength in HCS. The results show that interplanetary magnetic field lines are either turned around the MC body or they become reconnected across the HCS depending on the initial magnetic handedness of the MC.

The available simulation studies on the reconnections between MCs and HCS seem to be largely carried out in either $2 \mathrm{D}$ or $2.5 \mathrm{D}$. In this paper, we have used a $3 \mathrm{D}$ resistive $\mathrm{MHD}$ numerical simulation to study the reconnection process between the MC and HCS. When a MC moves to the HCS, the dynamic situation causes the current sheet to become gradually thinner and the magnetic reconnection process is initiated. We began by numerically forming a steady-state model of the heliospheric along with a reasonable large-scale structure of the solar wind near solar minimum. Then we superimpose a spherical plasmoid to mimic the MC, the reconnection take place when the MC moves to the HCS, we also show the 3D structure of the HCS in the reconnection process and compare different parameters which can effect the reconnection rate and the beginning time, such as the magnetic filed strengthen and direction in the $\mathrm{MC}$, the magnitude of velocity and direction in the $\mathrm{MC}$.

Before proceeding, let us note that we work in the framework of single-fluid MHD. Some justification of this approach is necessary given that the solar wind is known to undergo the transition from a collision-dominated to a weakly collisional plasma, meaning that different species not only can eventually acquire different temperatures but also can flow at different velocities (Riley et al. 2016; Cranmer et al. 2017; Gombosi et al. 2018). From the modeling perspective, even if neglecting less-abundant species such as alpha particles, and even if working with a fluid approach, one still needs to adopt separate energy equations for electrons and protons to address their vastly different behaviors in wave-particle interactions that are likely to heat the solar wind (Sturrock \& Hartle 1966; Hartle \& Sturrock 1968; Holzer \& Axford 1970; Marsch 1999, 2006; Hollweg \& Isenberg 2002). In the context of space weather, a multifluid approach has also been adopted to model the interactions of CMEs with the ambient solar wind (Jin et al. 2017a,b) within the framework of the Alfvén wave solar model.

The aim of the present paper is the large-scale process of magnetic reconnection in realistic interplanetary medium with spatial variations of all the solar wind parameters. For the time being, we have refrained from developing a multifluid MHD formulation in the consideration of selfconsistency.

First, the energy partition between electrons and protons is known to be difficult to handle self-consistently when shocks and/or turbulence are present, a point also recognized by Jin et al. (2017a). Shocks are inevitable in the present study. In the largely collisionless regime, shocks are associated with the intricate interplay between non-Maxwellian particle distributions and micro-instabilities that reach proton (e.g., ion-cyclotron) or even electron (e.g., whistlers) scales (Schwartz et al. 2013). Likewise, a rich variety of microinstabilities have been identified in the solar wind turbulence when one goes beyond the inertial range (Alexandrova et al. 2013). Wave-particle interactions at kinetic scales are expected to play an important role in determining the thermodynamics at fluid scales. However, the partition between different species of the energy acquisition due to shocks and/or turbulence is still an open issue. Part of our reason for adopting single-fluid MHD is that single-fluid MHD is capable of capturing the entropy increase across shock.

Second, heat conduction and viscosities are neglected due to the following considerations. It is well-known to be questionable to apply the classical Braginskii's formulae for these terms (Braginskii 1965) from the inner corona out to interplanetary space (Marsch 2006). Some studies that go beyond the Braginskii formulation tend to suggest that viscous heating seems to be less important than thermal conduction (Holzer et al. 1986), in agreement with the original suggestion by Parker et al. (1964). Regarding heat conduction, the proton one is substantially smaller than the electron one even in the collisiondominated regime (Braginskii 1965). In the collisionless case, the electron heat flux is expected to be limited by heat flux instabilities (Marsch 2006), and may not be far from the freestreaming limit (Hollweg 1976; Cranmer et al. 2009). Some previous numerical studies have incorporated the transition from the Spitzer law for the collision-dominated regime to Hollweg's formulation for the collisionless electron heat flux (Lionello et al. 2001; Endeve et al. 2003; Ofman 2004; Jin et al. 2017a). While somehow important in determining the electron temperature, this practice is found to have only a minor effect on the supersonic flow ( $\mathrm{Li}$ et al. 2005). Rather than following this practice, in the present study we have adopted a polytropic energy equation that partly addresses the effect of electron thermal conduction. The polytropic index was chosen to be 1.46 , in agreement with the value found from the Helios measurements of the ambient solar wind (Totten et al. 1995).

Third, it is recognized that a moment-based fluid approach can reproduce many aspects of collisionless magnetic reconnections, a ten-moment treatment seems necessary (Wang et al. 2015 , 2018). However, generalizing available studies to modeling the 3D solar wind on a global scale is numerically formidable. It is difficult to resolve small spatial scales associated with the Hall effect, let alone those small scales pertinent to electron inertia. We therefore have left this as a possible extension to our numerical scheme.

With the above-mentioned caveats in mind, our manuscript is organized as follows. In Sect. 2 MHD model in spherical coordinates and the simulation results for CR1915 solar wind background are described. Section 3 is devoted to the spherical plasmoid MC model. Section 4 introduces the simulation results of the reconnection process between the MC and HCS. Finally, some conclusions are given in Sect. 5 . 


\section{Model description}

In order to simulate the reconnection process between $\mathrm{MC}$ and HCS, a representative resistive MHD model of the steady state background solar wind is required. In this section, the 3D MHD simulation of the background solar wind in spherical coordinates for Carrington rotation (CR) 1915 is presented. The resistive MHD equations are splitted into the fluid part and the magnetic part, Harten-Lax-van Leer (HLL) scheme within a finite volume (FV) framework without Riemann solver or any kind of characteristic decomposition are used in this paper. To maintain the $\nabla \cdot \boldsymbol{B}=0$ constraint, the constraint transport (CT) technique is used for the magnetic field by utilizing a special discretization on a staggered grid (Ziegler 2004, 2011; Feng et al. 2014). The fluid part for the vector $\boldsymbol{U}=\left(\rho, \rho v_{\mathrm{r}}, \rho v_{\theta}, \rho v_{\phi} r \sin \theta, e\right)^{T}$ reads as follows

$$
\begin{aligned}
\frac{\partial \boldsymbol{U}}{\partial t} & +\frac{1}{r^{2}} \frac{\partial}{\partial r} r^{2}\left(\boldsymbol{F}-\boldsymbol{F}_{v}\right)+\frac{1}{r \sin \theta} \frac{\partial}{\partial \theta} \sin \theta\left(\boldsymbol{G}-\boldsymbol{G}_{v}\right) \\
& +\frac{1}{r \sin \theta} \frac{\partial}{\partial \phi}\left(\boldsymbol{H}-\boldsymbol{H}_{v}\right)=\boldsymbol{S}
\end{aligned}
$$

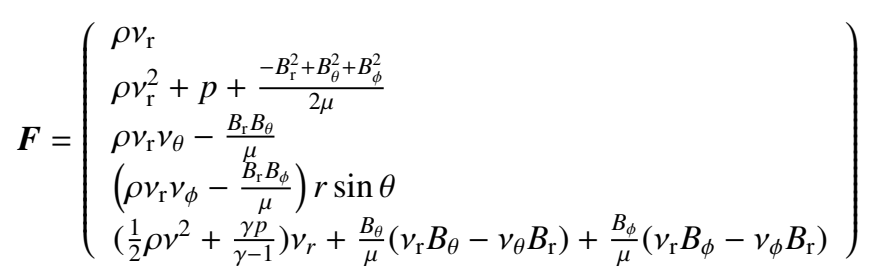$$
\boldsymbol{G}=\left(\begin{array}{l}
\rho v_{\theta} \\
\rho v_{\mathrm{r}} v_{\theta}-\frac{B_{\mathrm{r}} B_{\theta}}{\mu} \\
\rho v_{\theta}^{2}+p+\frac{B_{\mathrm{r}}^{2}-B_{\theta}^{2}+B_{\phi}^{2}}{2 \mu} \\
\left(\rho v_{\theta} v_{\phi}-\frac{B_{\theta} B_{\phi}}{\mu}\right) r \sin \theta \\
\left(\frac{1}{2} \rho v^{2}+\frac{\gamma p}{\gamma-1}\right) v_{\theta}+\frac{B_{\mathrm{r}}}{\mu}\left(v_{\theta} B_{\mathrm{r}}-v_{\mathrm{r}} B_{\theta}\right)+\frac{B_{\phi}}{\mu}\left(v_{\theta} B_{\phi}-v_{\phi} B_{\theta}\right)
\end{array}\right)
$$$$
\boldsymbol{H}=\left(\begin{array}{l}
\rho v_{\phi} \\
\rho v_{\mathrm{r}} v_{\phi}-\frac{B_{\mathrm{r}} B_{\phi}}{\mu} \\
\rho v_{\theta} v_{\phi}-\frac{B_{\theta} B_{\phi}}{\mu} \\
\left(\rho v_{\phi}^{2}+p+\frac{B_{\mathrm{r}}^{2}+B_{\theta}^{2}-B_{\phi}^{2}}{2 \mu}\right) r \sin \theta \\
\left(\frac{1}{2} \rho v^{2}+\frac{\gamma p}{\gamma-1}\right) v_{\phi}+\frac{B_{\mathrm{r}}}{\mu}\left(v_{\phi} B_{\mathrm{r}}-v_{\mathrm{r}} B_{\phi}\right)+\frac{B_{\theta}}{\mu}\left(v_{\phi} B_{\theta}-v_{\theta} B_{\phi}\right)
\end{array}\right)
$$$$
\boldsymbol{F}_{v}=\left(\begin{array}{l}
0 \\
0 \\
0 \\
0 \\
\frac{\eta}{\mu}\left(B_{\theta} j_{\phi}-B_{\phi} j_{\theta}\right)
\end{array}\right), \quad \boldsymbol{G}_{v}=\left(\begin{array}{l}
0 \\
0 \\
0 \\
0 \\
\frac{\eta}{\mu}\left(B_{\phi} j_{\mathrm{r}}-B_{\mathrm{r}} j_{\phi}\right)
\end{array}\right)
$$$$
\boldsymbol{H}_{v}=\left(\begin{array}{l}
0 \\
0 \\
0 \\
0 \\
\frac{\eta}{\mu}\left(B_{\mathrm{r}} j_{\theta}-B_{\theta} j_{\mathrm{r}}\right)
\end{array}\right)
$$$$
e=\frac{1}{2} \rho v^{2}+\frac{p}{\gamma-1}+\frac{1}{2 \mu} B^{2}, \quad j=\nabla \times B, \quad S=S_{1}+S_{2}
$$

with

$$
\boldsymbol{S}_{1}=\left(\begin{array}{l}
0 \\
\rho \frac{v_{\theta}^{2}+v_{\phi}^{2}}{r}+\frac{2 p}{r}+\frac{B_{\mathrm{r}}^{2}}{r \mu} \\
S_{1,3} \\
0 \\
0
\end{array}\right)
$$

$$
\boldsymbol{S}_{2}=\left(\begin{array}{l}
0 \\
\rho \Omega \sin \theta\left(2 v_{\phi}+\Omega r \sin \theta\right) \\
\rho \Omega \cos \left(2 v_{\phi}+\Omega r \sin \theta\right) \\
-2 \rho \Omega\left(v_{\theta} \cos \theta+v_{\mathrm{r}} \sin \theta\right) r \sin \theta \\
\left.\rho v_{\mathrm{r}} \Omega^{2} r \sin ^{2} \theta+\rho v_{\theta} \Omega^{2} r \sin \theta \cos \theta\right)
\end{array}\right)
$$

where

$$
S_{1,3}=\left(p+\rho v_{\phi}^{2}+\frac{B_{\mathrm{r}}^{2}+B_{\theta}^{2}-B_{\phi}^{2}}{2 \mu}\right) \frac{\cot \theta}{r}+\frac{1}{r}\left(\frac{B_{\theta} B_{\mathrm{r}}}{\mu}-\rho v_{\mathrm{r}} v_{\theta}\right)
$$

$\eta$ is the electric resistivity, $\boldsymbol{j}$ is the current density, $e$ corresponds to the total energy density consisting of the kinetic, thermal, and magnetic energy density. $\rho$ is the mass density, $v=\left(v_{\mathrm{r}}, v_{\theta}, v_{\phi}\right)$ are the flow velocity in the frame rotating with the Sun, $p$ is the thermal pressure. $t$ and $\boldsymbol{r}$ are time and position vectors originating at the center of the Sun. $\mu=4 \times 10^{-7} \pi, \gamma=1.46$, and $|\Omega|=2 \pi / 26.3 \mathrm{rad} \mathrm{day}^{-1}$ is the solar angular speed.

In the above formulas, the first source term $S_{1}=$ $\left(S_{1,1}, \ldots, S_{1,5}\right)^{T}$ arises from the polar geometrical factors, while the second source term $S_{2}=\left(S_{2,1}, \ldots, S_{2,5}\right)^{T}$ does due to the Coriolis, centrifugal.

The subsystem for magnetic induction part runs as follows

$$
\begin{aligned}
\frac{\partial B_{\mathrm{r}}}{\partial t} & +\frac{1}{r \sin \theta} \frac{\partial}{\partial \theta}\left(\sin \theta\left(v_{\theta} B_{\mathrm{r}}-v_{\mathrm{r}} B_{\theta}\right)\right)-\frac{1}{r \sin \theta} \frac{\partial}{\partial \phi}\left(v_{\mathrm{r}} B_{\phi}-v_{\phi} B_{\mathrm{r}}\right) \\
& =\frac{\eta}{r \sin \theta} \frac{\partial}{\partial \phi} j_{\theta}-\frac{\eta}{r \sin \theta} \frac{\partial}{\partial \theta}\left(\sin \theta j_{\phi}\right) \\
\frac{\partial B_{\theta}}{\partial t} & -\frac{1}{r} \frac{\partial}{\partial r}\left(r\left(v_{\theta} B_{\mathrm{r}}-v_{\mathrm{r}} B_{\theta}\right)\right)+\frac{1}{r \sin \theta} \frac{\partial}{\partial \phi}\left(v_{\phi} B_{\theta}-v_{\theta} B_{\phi}\right) \\
& =\frac{\eta}{r} \frac{\partial}{\partial r}\left(r j_{\phi}\right)-\frac{\eta}{r \sin \theta} \frac{\partial}{\partial \phi} j_{\mathrm{r}} \\
\frac{\partial B_{\phi}}{\partial t} & +\frac{1}{r} \frac{\partial}{\partial r}\left(r\left(v_{\mathrm{r}} B_{\phi}-v_{\phi} B_{\mathrm{r}}\right)\right)-\frac{1}{r} \frac{\partial}{\partial \theta}\left(v_{\phi} B_{\theta}-v_{\theta} B_{\phi}\right) \\
& =\frac{\eta}{r} \frac{\partial}{\partial \theta} j_{\mathrm{r}}-\frac{\eta}{r} \frac{\partial}{\partial r}\left(r j_{\theta}\right)
\end{aligned}
$$

As usual, $\rho, v, p, \boldsymbol{B}, r, \eta$, and $t$ are normalized by the characteristic values $\rho_{\mathrm{s}}, a_{\mathrm{s}}, \rho_{\mathrm{s}} a_{\mathrm{s}}^{2}, \sqrt{\mu \rho_{\mathrm{s}} a_{\mathrm{s}}^{2}}, R_{\mathrm{s}}, \mu R_{\mathrm{s}} a_{\mathrm{s}}$ and $R_{\mathrm{s}} / a_{\mathrm{s}}$, where $\rho_{\mathrm{s}}, a_{\mathrm{s}}$ are the density and sound speed at the solar surface. $R_{\mathrm{S}}=$ $6.9 \times 10^{8} \mathrm{~m}$ is the solar radius.

The computational domain here covers $20 R_{\mathrm{s}} \leq r \leq 110 R_{\mathrm{s}}$, $45^{\circ} \leq \theta \leq 135^{\circ}$ and $-45^{\circ} \leq \phi \leq 45^{\circ}$, where $r$ is the radial distance from solar center in units of solar radius $R_{\mathrm{s}}, \theta$ is latitude angle and $\phi$ is the longitude angle, respectively. Because the MC observed at earth usually occurred at the vicinity of HCS, and we are only concerned with the theory of the process of interaction between MC and HCS, so the computational domain in our code covers only a part of the whole heliosphere. The grid mesh is chosen to be $1000(r) \times 390(\theta) \times 180(\phi)$. The grid size is uniform in radial with $\Delta r=0.08 R_{\mathrm{s}}$ and azimuth with $\Delta \phi=0.5^{\circ}$. The meridional grids $\Delta \theta(j)$ are not uniform, in order to obtain a 
precise computational resolution near HCS. The smallest grid is near HCS is $\Delta \theta=0.0625^{\circ}$ and the biggest grid far from the HCS is $\Delta \theta=1^{\circ}$.

The numerical scheme we used is a 3D HLL scheme within a FV framework following Feng et al. (2014) by splitting these equations into a fluid part Eq. (1) and a magnetic part Eqs. (2)-(4). For spatial discretization, we used the FV discretization of Eq. (1), and average Eqs. (2)-(4) over facial areas to get semi-integral form of magnetic induction equations. Second-order accurate linear ansatz reconstruction is adopted for the fluid part, and the derivative terms were approximated by using the minmod limiter for oscillation control. We used the CT method for the magnetic induction equations. For consistency, the reconstruction for magnetic induction part is also of second-order accuracy and cross derivative terms are slopelimited approximations to the exact derivatives at cell face centers by using minmod limiter. The full system is integrated in time with a second-order Runge-Kutta scheme. For details, refer to Feng et al. (2014). Since the HLL scheme only captures the two fastest waves accurately and its resolution for the other waves are therefore insufficient, we used the HLL numerical flux in the fluid part plus some antidiffusion terms in this paper to improve the overall efficiency of the scheme. We introduced antidiffusion terms for all but the two fast waves, take the interface numerical flux in radial direction $\tilde{\boldsymbol{F}}$ for example:

$\tilde{\boldsymbol{F}}\left(\boldsymbol{U}_{\mathrm{L}}, \boldsymbol{U}_{\mathrm{R}}\right)=\tilde{\boldsymbol{F}}^{\mathrm{HLL}}\left(\boldsymbol{U}_{\mathrm{L}}, \boldsymbol{U}_{\mathrm{R}}\right)-\varphi \boldsymbol{a}_{0}\left(\boldsymbol{U}_{\mathrm{L}}, \boldsymbol{U}_{\mathrm{R}}\right)$

where $\tilde{\boldsymbol{F}}^{\mathrm{HLL}}\left(\boldsymbol{U}_{\mathrm{L}}, \boldsymbol{U}_{\mathrm{R}}\right)$ denotes the numerical flux of the standard HLL scheme, $\boldsymbol{U}_{\mathrm{L}}$ and $\boldsymbol{U}_{\mathrm{R}}$ are the left and right hand states, respectively, of a local Riemann problem, and $\boldsymbol{U}_{\mathrm{M}}=\frac{\boldsymbol{U}_{\mathrm{L}}+\boldsymbol{U}_{\mathrm{R}}}{2}$ is their arithmetic average.

$\tilde{\boldsymbol{F}}^{\mathrm{HLL}}\left(\boldsymbol{U}_{\mathrm{L}}, \boldsymbol{U}_{\mathrm{R}}\right)=\frac{c_{\mathrm{R}} \boldsymbol{F}\left(\boldsymbol{U}_{\mathrm{L}}\right)-c_{\mathrm{L}} \boldsymbol{F}\left(\boldsymbol{U}_{\mathrm{R}}\right)}{c_{\mathrm{R}}-c_{\mathrm{L}}}+\frac{c_{\mathrm{R}} c_{\mathrm{L}}}{c_{\mathrm{R}}-c_{\mathrm{L}}}\left(\boldsymbol{U}_{\mathrm{R}}-\boldsymbol{U}_{\mathrm{L}}\right)$

$c_{\mathrm{L}}=\min \left\{\left(v_{\mathrm{r}}-c_{\mathrm{fr}}\right)_{\mathrm{L}},\left(v_{\mathrm{r}}-c_{\mathrm{fr}}\right)_{\mathrm{R}}, 0\right\}$

$c_{\mathrm{R}}=\max \left\{\left(v_{\mathrm{r}}+c_{\mathrm{fr}}\right)_{\mathrm{L}},\left(v_{\mathrm{r}}+c_{\mathrm{fr}}\right)_{\mathrm{R}}, 0\right\}$

where, $c_{\mathrm{fr}}=\frac{1}{\sqrt{2}} \sqrt{u_{\mathrm{s}}^{2}+u_{\mathrm{A}}^{2}+\left(\left(u_{\mathrm{s}}^{2}+u_{\mathrm{A}}^{2}\right)^{2}-4 u_{\mathrm{s}}^{2} \frac{B_{\mathrm{r}}^{2}}{\mu \rho}\right)^{\frac{1}{2}}}$ is the fast magnetosonic speed in radial direction. $u_{\mathrm{s}}=\sqrt{\frac{\gamma p}{\rho}}, u_{\mathrm{A}}=$ $\sqrt{\frac{B_{\mathrm{r}}^{2}+B_{\theta}^{2}+B_{\phi}^{2}}{\mu \rho}}$ are sound speed and Alfvénic speed, respectively. The antidiffusion term

$\boldsymbol{a}_{0}\left(\boldsymbol{U}_{\mathrm{L}}, \boldsymbol{U}_{\mathrm{R}}\right)=\frac{c_{\mathrm{R}} c_{\mathrm{L}}}{c_{\mathrm{R}}-c_{\mathrm{L}}} \sum_{i=2}^{6} \delta_{i} \alpha_{i} \boldsymbol{r}_{i}\left(\boldsymbol{U}_{\mathrm{M}}\right)$

In the MHD case the Jacobian matrix $\frac{\partial \boldsymbol{F}}{\partial \boldsymbol{U}}$ has seven eigenvalues $\lambda_{1}(\boldsymbol{U}) \leq \cdots \leq \lambda_{7}(\boldsymbol{U}) . \boldsymbol{l}_{i}\left(\boldsymbol{U}_{\mathrm{M}}\right), \boldsymbol{r}_{i}\left(\boldsymbol{U}_{\mathrm{M}}\right)$ are the left and right eigenvectors of eigenvalues $\lambda_{i}\left(\boldsymbol{U}_{\mathrm{M}}\right)$, respectively. The coefficients $\alpha_{i}$ result from the decomposition of $\boldsymbol{U}_{\mathrm{R}}-\boldsymbol{U}_{\mathrm{L}}$ into the contributions of the right eigenvectors $\boldsymbol{r}_{i}$, i.e., $\boldsymbol{U}_{\mathrm{R}}-\boldsymbol{U}_{\mathrm{L}}=\sum_{i=1}^{7} \alpha_{i} \boldsymbol{r}_{i}\left(\boldsymbol{U}_{\mathrm{M}}\right)$. The antidiffusion coefficients $\delta_{2}=\delta_{6}=\delta_{a}, \delta_{3}=\delta_{5}=\delta_{s}, \delta_{4}=\delta_{\mathrm{e}}$ read

$\delta_{\mathrm{e}}=\frac{c_{\mathrm{fr}}\left(\boldsymbol{U}_{\mathrm{M}}\right)}{c_{\mathrm{fr}}\left(\boldsymbol{U}_{\mathrm{M}}\right)+\left|v_{\mathrm{r}}\left(\boldsymbol{U}_{\mathrm{M}}\right)\right|}$

$\delta_{\mathrm{s}}=\frac{c_{\mathrm{fr}}\left(\boldsymbol{U}_{\mathrm{M}}\right)}{c_{\mathrm{fr}}\left(\boldsymbol{U}_{\mathrm{M}}\right)+c_{\mathrm{sr}}\left(\boldsymbol{U}_{\mathrm{M}}\right)}$
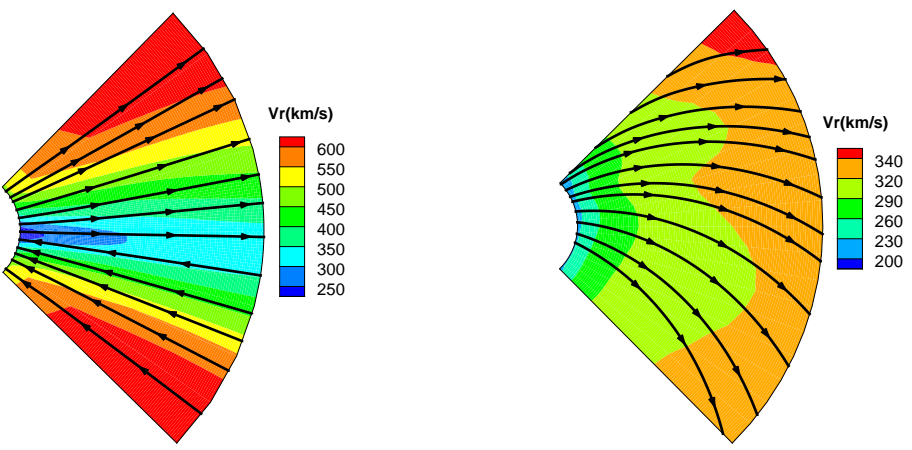

Fig. 1. Magnetic field and radial speed $v_{\mathrm{r}}\left(\mathrm{km} \mathrm{s}^{-1}\right)$ for the steady state solar wind solution. Left: on the meridional plane of $\phi=0^{\circ}$ from 20 to $110 R_{\mathrm{s}}$, right: on the equatorial plane. The color contours represent the radial speed and streamlines denote the magnetic field lines.

$\delta_{\mathrm{e}}=\frac{c_{\mathrm{fr}}\left(\boldsymbol{U}_{\mathrm{M}}\right)}{c_{\mathrm{fr}}\left(\boldsymbol{U}_{\mathrm{M}}\right)+c_{\mathrm{ar}}\left(\boldsymbol{U}_{\mathrm{M}}\right)}$

where $c_{\mathrm{sr}}=\frac{1}{\sqrt{2}} \sqrt{u_{\mathrm{s}}^{2}+u_{\mathrm{A}}^{2}-\left(\left(u_{\mathrm{s}}^{2}+u_{\mathrm{A}}^{2}\right)^{2}-4 u_{\mathrm{s}}^{2} \frac{B_{\mathrm{r}}^{2}}{\mu \rho}\right)^{\frac{1}{2}}}, c_{\mathrm{ar}}=\frac{\left|B_{\mathrm{r}}\right|}{\mu \rho}$. Moreover, we set $\varphi=\Phi\left(\beta_{\mathrm{r}}\right)=\Phi\left(\frac{2 u_{\mathrm{s}}^{2}}{\gamma c_{\mathrm{ar}}^{2}}\right)$. In the above formulation, $\Phi$ is the Lipschitz-continuous switch function defined by

$\begin{cases}x \in\left(-\infty, \epsilon_{1}\right) & \Phi(x)=0 \\ x \in\left[\epsilon_{1}, \delta_{1}\right) & \Phi(x)=\frac{x-\epsilon_{1}}{\delta_{1}-\epsilon_{1}} \\ x \in\left[\delta_{1}, \infty\right) & \Phi(x)=1\end{cases}$

with $\epsilon_{1}=\frac{2}{\gamma}, \delta_{1}=2 \epsilon_{1}, \varphi$ is required to maintain the conservation property of the scheme as stated in Lemma 4.1 of Wesenberg (2010). According to the same lemmas, if $B_{\mathrm{r}} \neq 0, B_{\theta}^{2}+B_{\phi}^{2}=0$, and $u_{\mathrm{s}}^{2}-c_{\mathrm{ar}}^{2}<0$, set $\varphi=0$, which means that the antidiffusion is switched off, since in this case the eigenvectors and coefficients contributing to a swap to wrong positions, and thus the conservation property is lost. The definition of $\varphi$ implies that the antidiffusion is switched off in small plasma $\beta_{\mathrm{r}}$ region.

Here, we present the steady state solar wind numerical results from the 3D MHD numerical simulation for CR 1915, Fig. 1 shows the MHD results for the magnetic fields and radial speed for the steady state solar wind solution, The left is on the meridional plane of $\phi=0^{\circ}$ from 20 to $110 R_{\mathrm{s}}$, and the right is on the equatorial plane, the arrowheads denote the direction of the magnetic field and the color contours represent the radial solar wind speed. From this picture, it is seen that the magnetic field and radial speed possess a typical characteristic of solar minimum. The magnetic field lines extend to interplanetary space, and there appear fast solar wind flows at high latitudes and the slow solar wind are showed in the vicinity of the equator or HCS region. The solar wind extends the interplanetary magnetic field outward into Archimedean spirals due to the solar rotation and the interplanetary magnetic field freezing-in effect.

\section{Spheromak MC model}

In this paper, a very simple spherical plasmoid model is superposed on the background solar wind to initiate the MC, which contains a high speed, high density and high pressure spherical plasmoid (Zhou et al. 2012; Zhou \& Feng 2013). This kind of model do not have very complex features and gives us an easy 
way to study the evolution of the MC in the interplanetary space. The density, pressure, and velocity profile of the initial perturbation are defined as follows

$\rho=\rho_{0}+\rho_{\max }\left(1-\frac{a^{2}}{a_{\mathrm{mc}}^{2}}\right)$

$v=v_{0}+v_{\max }\left(1-\frac{a^{2}}{a_{\mathrm{mc}}^{2}}\right)$

$p=p_{0}+p_{\max }\left(1-\frac{a^{2}}{a_{\mathrm{mc}}^{2}}\right)$

where $a_{\mathrm{mc}}$ is the radius of the plasmoid, $a$ denotes the distance from the center of the plasmoid, and $\rho_{0}, v_{0}$ and $p_{0}$ are the density, velocity, and pressure of the background solar wind, respectively. $\rho_{\max }, v_{\max }$ and $p_{\max }$ are the maximum density, velocity and pressure added on top of the background solar wind, respectively. The choices of these parameters are empirical. In this paper, $\rho_{\max }$ is assumed to have a higher valve than the background solar wind by a factor of two. Since CMEs measured by Ulysses show different speeds in the solar wind, $v_{\max }$ can be assumed to move with respect to the ambient solar wind at a range of different speeds (Plunkett \& Wu 2000; Schmidt \& Cargill 2001, 2003), the direction of $v_{\max }$ is along the negative $z$ axis in this paper. $p_{\max }$ is assumed to be six of the ambient solar wind at the center of the CME, observations from the Ulysses spacecraft shows that such pressure enhancements may be a common feature in MCs near the Sun (Gosling et al. 1994; Schmidt \& Cargill 2001, 2003).

A spheromak-type magnetic field which is a linear forcefree field in a completely isolated sphere is introduced as the simplest MC model in this paper (Kataoka et al. 2009). The actual existence of magnetic field in the solar wind is under debate, but Gibson \& Fan (2008) believe that the spheromaktype magnetic field may be a natural product of a plasmoid ejected from a solar flare via reconnection. Wang et al. (2010a,b) also described certain existence evidence on plasmoid in the magneto tail. In the present paper, the initial shape of the MC is assumed to be a sphere with the radius $a_{\mathrm{mc}}$ in local spherical coordinates $(\tilde{r}, \tilde{\theta}, \tilde{\phi})$

$\tilde{B}_{\tilde{\mathrm{r}}}(\tilde{r}, \tilde{\theta}, \tilde{\phi})=\left(2 B_{0} / \alpha \tilde{r}\right) j_{1}(\alpha \tilde{r}) \cos \tilde{\theta}$

$\tilde{B}_{\tilde{\theta}}(\tilde{r}, \tilde{\theta}, \tilde{\phi})=-\left(B_{0} / \alpha \tilde{r}\right)\left[\sin (\alpha \tilde{r})-j_{1}(\alpha \tilde{r})\right] \sin \tilde{\theta}$

$\tilde{B}_{\tilde{\phi}}(\tilde{r}, \tilde{\theta}, \tilde{\phi})= \pm B_{0} j_{1}(\alpha \tilde{r}) \sin \tilde{\theta}$

where $B_{0}$ is a constant means the field strength of the spheromak. $j_{1}$ is the first-order spherical Bessel function

$j_{1}(x)=\frac{\sin x-x \cos x}{x^{2}}$

$\alpha=4.493409458 a_{\mathrm{mc}}$ is the constant derived from the force-free condition of $\nabla \times \boldsymbol{B}=\alpha \boldsymbol{B}$ with the boundary condition of $\tilde{B}_{\tilde{\mathrm{r}}}=0$ at $\tilde{r}=a_{\mathrm{mc}}$, we adopted only the field within the isolated spherical shell $\tilde{r} \leq a_{\mathrm{mc}}$. In this paper, we chose the position of the center of the spheromak at $\left(30 R_{\mathrm{s}}, 80^{\circ}, 0^{\circ}\right)$ in spherical coordinates. The radius of the initial MC, $a_{\mathrm{mc}}$, is $6 R_{\mathrm{s}}$. The maximal magnetic field strength $B_{0}$ is $500 \mathrm{nT}$. Although a spheromak is a linear forcefree field, we adopted it only within the boundary $\tilde{r} \leq a_{\mathrm{mc}}$. At the spherical boundary $\tilde{r}=a_{\mathrm{mc}}$, the local radial $\tilde{B}_{\tilde{\mathrm{r}}}$ and azimuthal $\tilde{B}_{\tilde{\phi}}$ components vanish and only the local zenithal component $\tilde{B}_{\tilde{\theta}}$

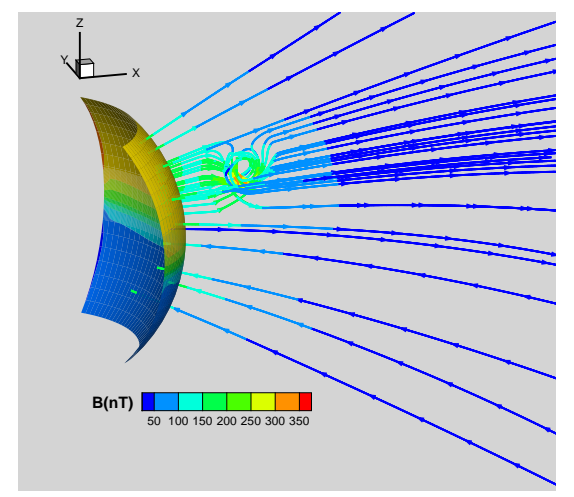

Fig. 2. Three-dimensional view of the initial magnetic field. Field lines are shown in color to illustrate the magnetic field strength. The color contours represent the radial magnetic field strength on the surface at $r=20 R_{\mathrm{s}}$.

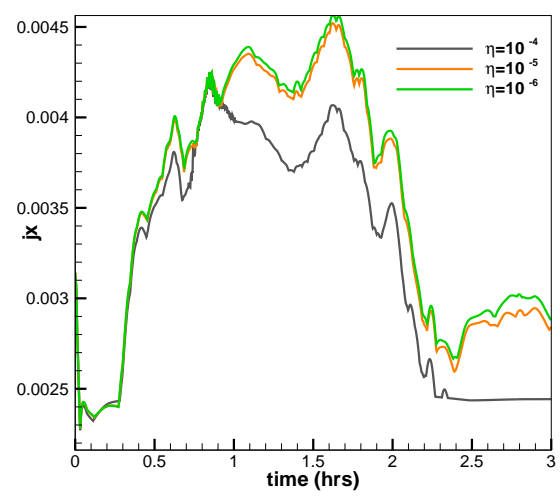

Fig. 3. Profile of maximum current density $j_{x}$ with time, in which the black line represents $\eta=10^{-4}$, the orange line represents $\eta=10^{-5}$ and the green line is $\eta=10^{-6}$.

exists, while $\tilde{B}=0$ outside of the boundary. Therefore, the forces do not balance at the spherical boundary. The spheromak field naturally swells in the simulation (Shiota et al. 2010). Figure 2 shows the 3D schematic picture of the initial magnetic field at $t=0$. Field lines are shown in color to illustrate the magnetic field strength. The color contours represent the radial magnetic field strength on the surface at $r=20 R_{\mathrm{s}}$.

\section{Results and discussion}

In this section, we present the numerical results for the reconnection process between MC and HCS. Magnetic reconnection is a main concern in this paper and magnetic reconnection simulation needs very high numerical resolution. If the numerical diffusion is large, then the real physical reconnection process will be hide. Before experiments commence, it is necessary to roughly estimate the numerical diffusion compared in our code.

The flux rope was initially assumed to move at four times the solar wind speed. Figure 3 gives the profile of maximum current density $j_{x}$ with time when a MC moves to HCS, we tested different magnetic diffusivity coefficients, in which the black line represents $\eta=10^{-4}$, the orange line represents $\eta=10^{-5}$ and the green line is $\eta=10^{-6}$. For $\eta=10^{-4}$, since the MC moving toward the current sheet, the current density $j_{x}$ increase soon after the beginning of simulation. After $t=0.5 \mathrm{~h}$, the current sheet becomes thinner in the region impacted by the plasma blob and the reconnection take place. The evolution of the maximum 

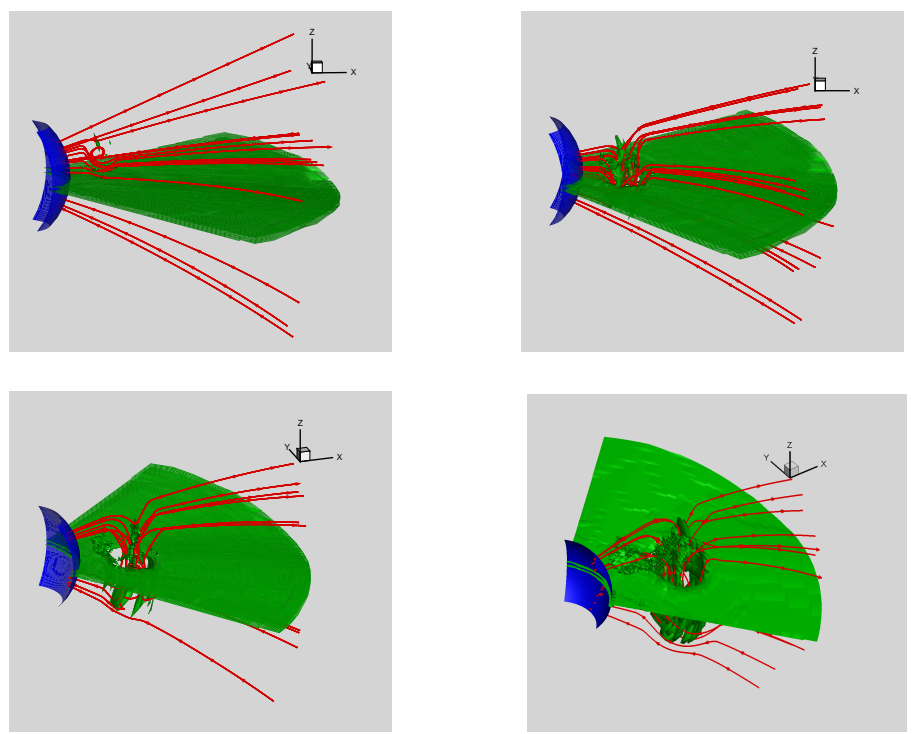

Fig. 4. 3D representation of the MC is shown at zero hours (top left), three hours (top right), six hours (bottom left), and $12 \mathrm{~h}$ (bottom right). The HCS is approximated by a green surface. The streamlines showed in red denote the magnetic field lines.

current density $j_{x}$ in the case of $\eta=10^{-5}$ is similar to that in the case $\eta=10^{-4}$ during the initial phase, however, the current density $j_{x}$ becomes larger after $t=1 h$ compared to $\eta=10^{-4}$. There are little difference between $\eta=10^{-6}$ and $\eta=10^{-5}$. This suggests that effective numerical diffusion is on the order of $5 \times 10^{-5}$ in our code.

Figure 4 shows a 3D view of the magnetic field at zero hours, three hours, six hours, and $12 \mathrm{~h}$. The green surface shows the evolution of the HCS during the reconnection. The streamlines showed in red denote the magnetic field lines. The HCS is defined as a iso-surface of $\frac{|\nabla \times \boldsymbol{B}|}{|\boldsymbol{B}|}$. The MC located above the current sheet at $t=0 \mathrm{~h}$, and the direction of the magnetic field in $\mathrm{MC}$ is anticlockwise. From this figure, we find that initially the magnetic field evolves nearly in self-similar manner. The plasmoid quickly expands because of the higher plasma density, speed, and magnetic pressure in the MC. The radial and angular sizes of the MC increase with time. As the MC moving toward the current sheet, the current sheet becomes thinner and reconnection between the $\mathrm{MC}$ and solar wind takes place since they have antiparallel magnetic lines. The reconnection take place mainly at leading edge of the flux rope, which is a driven reconnection. The HCS is deformed and bent during reconnection, which can be seen clearly at three hours, six hours, and $12 \mathrm{~h}$. We also find that reconnection takes place at the rear edge of the flux rope. We cannot reach a high enough accuracy because of the computation, so this is a Sweet-Parker reconnection. We also find a local current sheet inside the $\mathrm{MC}$ in this figure.

Figure 5 shows magnetic reconnection driven by the MC moving toward the current sheet on the meridional plane, the model results for the relative density $\frac{\rho-\rho_{0}}{\rho_{0}}$ and current density $j_{\phi}$ at different times. $j_{\phi}$ denotes the out-of-plane component of the current density. The reconnection appears to be primarily located at the leading edge (southward) of the flux rope. We see obvious single X-line reconnections begin to develop in the region of the current sheet, where the relative density and current density are bigger. These results are caused by the strong driving together of oppositely directed field lines at the leading edge of the MC due
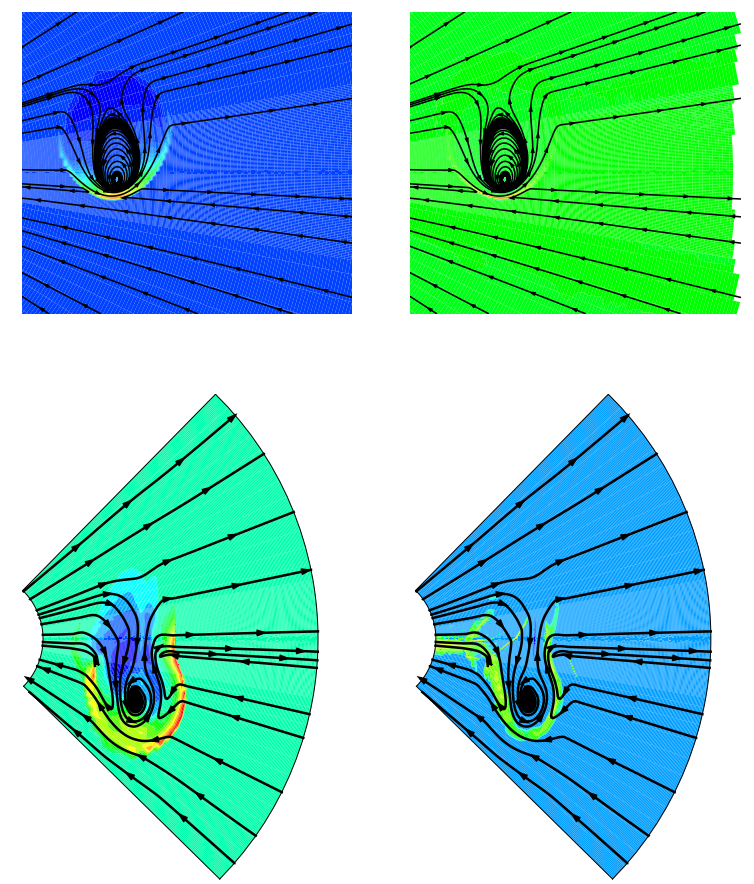

Fig. 5. Magnetic reconnection driven by the MC moving toward the current sheet on the meridional plane, top row: model results for the relative density $\frac{\rho-\rho_{0}}{\rho_{0}}$ (left) and current density $j_{\phi}$ (right) at $t=1 \mathrm{~h}$, bottom row: $t=9 h$. The streamlines denote the magnetic field lines.
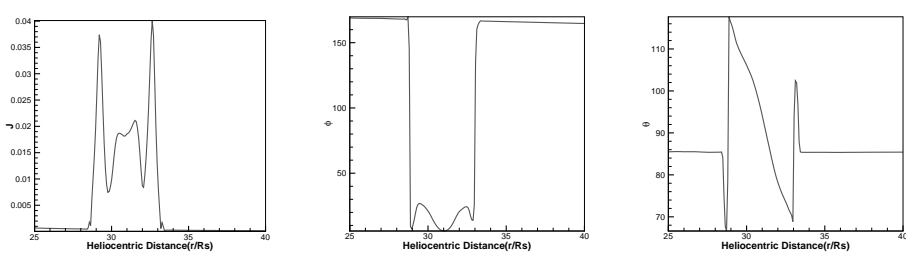

Fig. 6. Numerical results of the current density, the change of azimuth and the latitude angle of the magnetic field along heliocentric distance with $\theta=95^{\circ}$ and $\phi=0^{\circ}$ for $t=1 h$.

to its relative motion with respect to the solar wind. It also can be seen from the figure that the current sheet is being compressed and a bow region with high density appears. The supersonic flow generates a fast mode shock and a sheath ahead of the MC. At the shock front, the solar wind plasma becomes compressed and heated. This is clearly visible according to the enhancements of density.

Figure 6 shows the variations of the basic parameters versus the distance, which would be recorded by an observer if it passed through the numerical test region of the magnetic reconnection along $\theta=95^{\circ}$ and $\phi=0^{\circ}$ for $t=1 \mathrm{~h}$. It can be seen clearly that the two increasing of the current density, respectively, located at $r=29 R_{\mathrm{s}}$ and $r=33 R_{\mathrm{s}}$, where the correlative variations also are simultaneously recorded, such as about $170^{\circ}$ variation of the azimuthal direction for the magnetic field, $50^{\circ}$ variation of the latitude direction. These basic features imply that the magnetic reconnections occur in the regions located at $r=29 R_{\mathrm{s}}$ and $r=$ $33 R_{\mathrm{s}}$ (Wei et al. 1997, 2005).

If a spacecraft passes through the numerical test region of the magnetic reconnection along $x$ direction on the plane of $\phi=0^{\circ}$ at $t=6 \mathrm{~h}$, it will find a reconnection exhaust region. Figure 7 shows the magnetic field components, flow velocity components, proton number density, the proton temperature, and 

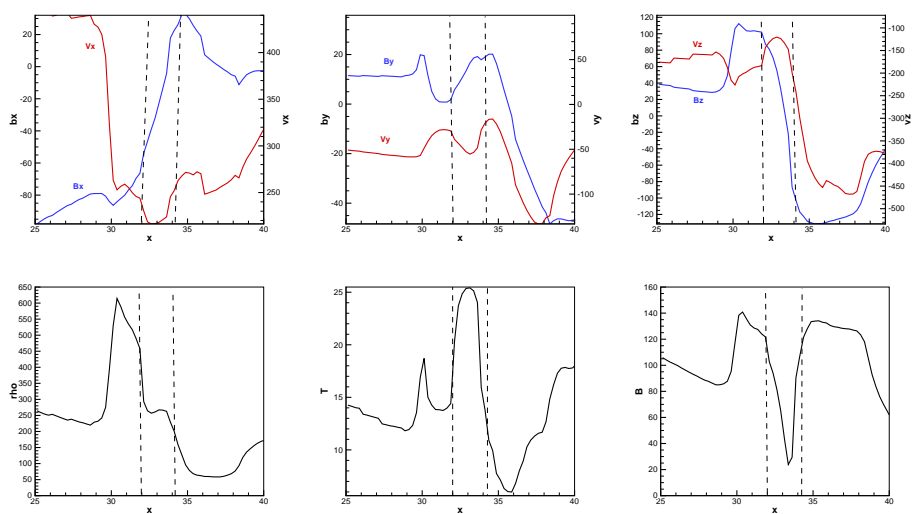

Fig. 7. Selected plasma and magnetic field data along $x$ direction on the meridional plane at $t=6 \mathrm{~h}$. Top row: magnetic field and flow velocity components in $(x, y, z)$ coordinates. Bottom row: proton number density (left), proton temperature (middle) and magnetic field strength (right). Vertical lines bracket a reconnection exhaust.

the magnetic field strength along $x$ direction with $x=[25,40]$, $z=-11$ on the plane of $\phi=0^{\circ}$ at $t=6 \mathrm{~h}$. Vertical lines bracket a reconnection exhaust. Since Alfvén waves propagating parallel (antiparallel) to $\boldsymbol{B}$ produce anticorrelated (correlated) variations in $\boldsymbol{B}$ and $\boldsymbol{V}$, one expects to observe anticorrelated changes in $\boldsymbol{B}$ and $\boldsymbol{V}$ at the leading edge of the field reversal region and correlated variations in $\boldsymbol{B}$ and $\boldsymbol{V}$ at the trailing edge in Fig. 7. Such pairs of coupled changes in $\boldsymbol{B}$ and $\boldsymbol{V}$ are the characteristic signature by which we identify reconnection exhausts in the solar wind (Gosling et al. 2007; Gosling 2008; Gosling \& Phan 2013; Wang et al. 2010c). The exhaust was associated with a small increase in proton temperature and a decrease in magnetic field strength, there has a decrease in the proton number densities within the exhaust. The result differed from most reconnection exhausts observed in the solar wind in that no clear increases in proton number density was detected within the exhaust. Helios, ACE, and Ulysses data also show similar density signals. This indicates that the transitions from outside to inside reconnection exhausts in the solar wind are not always slowmode-like, even though most such transitions are (Gosling et al. 2006a).

Figure 8 shows the velocity and magnetic field data for Fig. 7 are plotted in an exhaust-oriented coordinate system $(L, M, N)$ derived from minimum variance analysis of the filtered magnetic field data. The magnetic field across the exhaust with a nearly constant zero normal field $B_{N}$ and an entirely reversed antiparallel field $B_{L}$. The velocity $V_{L}$ in the exhaust $\left(\Delta V_{L}=70 \mathrm{~km} \mathrm{~s}^{-1}\right)$ was just consistent with the Alfvén reconnection jet, and the small normal velocity shear $\left(\Delta V_{N}=10 \mathrm{~km} \mathrm{~s}^{-1}\right)$ across bifurcated current sheet could be considered as a $5 \mathrm{~km} \mathrm{~s}^{-1}\left(V_{\text {in }}=\right.$ $\left.\Delta V_{N} / 2\right)$ reconnection inflow. Correspondingly, the dimensionless reconnection rates were calculated to be 0.07 (Wang et al. 2010c).

We ran a case in which the flux rope has an opposite sense of rotation such that the direction of the magnetic field in MC is clockwise. In this case, the positions at which the reconnection take place are different compared to the above case that the magnetic field in MC is anticlockwise. Figure 9 shows magnetic reconnection driven by the MC moving toward the current sheet on the meridional plane, the model results for the magnetic fields lines at different times. The reconnection appears to be primarily located at the leading edge (southward) of the flux rope soon after the beginning of simulation, since the magnetic field lines between the MC and the solar wind are antiparallel, the MC is
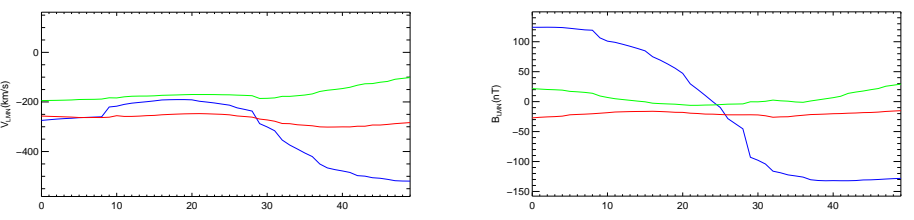

Fig. 8. Velocity $(l e f t)$ and magnetic field $(r i g h t)$ data are plotted in an exhaust-oriented coordinate system $(L, M, N)$ derived from minimum variance analysis of the filtered magnetic field data. Here $L$ (blue), $M$ (green), and $N$ (red) correspond to the maximum, intermediate, and minimum variance directions, respectively.
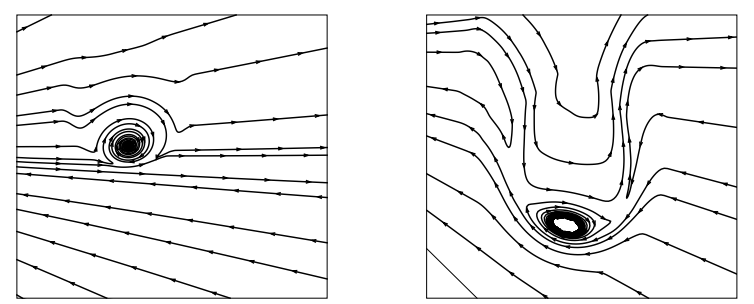

Fig. 9. Magnetic reconnection driven by the MC moving toward the current sheet on the meridional plane, the model results for the magnetic fields lines at $t=0.5 h$ (left) and $t=6 h($ right).
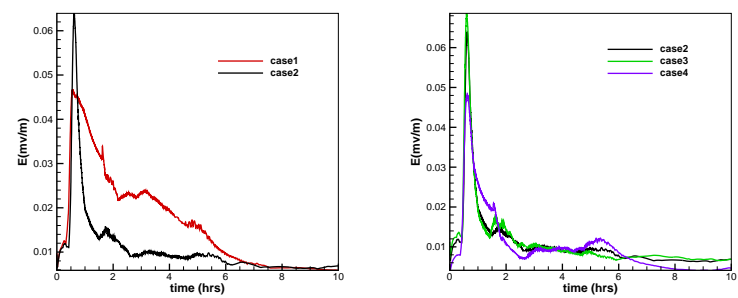

Fig. 10. Left: reconnection electric field $E\left(\mathrm{mv} \mathrm{m}^{-1}\right)$ as a function of time for a MC moving with different magnetic fields direction: Case1 (anticlockwise), Case2 (clockwise). Right: different magnetic field strengths: Case2 (500 nT), Case3 (600 nT), Case4 (300 nT).

eroded at the front, which can be clearly seen at $t=0.5 \mathrm{~h}$ in Fig. 9. As the MC moves to the HCS, the magnetic field lines between the MC and the solar wind at the leading edge of the flux rope become parallel, and the reconnection terminate, which can be clearly seen at $t=6 \mathrm{~h}$. At the same time, we find reconnection takes place at two sides of the flux rope, which can be interpreted as the fluid behind the MC squeeze the MC and arouse the driven reconnection (Wei et al. 2002).

Figure 10 shows the reconnection electric field $E\left(\mathrm{mv} \mathrm{m}^{-1}\right)$ as a function of time for a MC moving with the magnetic fields for the following cases: For Case 1 and Case2, the $B_{0}$ are $500 \mathrm{nT}$, but the direction of the magnetic field in $\mathrm{MC}$ are different; for Case2, Case3, and Case4, the direction of the magnetic field in $\mathrm{MC}$ are the same, but the $B_{0}$ are different. The reconnection electric field is measured by $\max \left(\eta j_{\phi}\right)$ (Birn \& Hesse 2001; Schmidt \& Cargill 2003; Feng et al. 2006; Borovsky et al. 2008; Zhang et al. 2011). We find the reconnection electric field for the Case 2 is larger than for Case 1 , and the reconnection electric field is depend on the direction of the magnetic field. For Case2, Case3, and Case4, the magnetic fields strengthen in MC are different, the reconnection electric field for Case 3 is apparently larger than for Case 2 and Case 4. So the reconnection electric field is also depend on the magnetic fields strengthen in MC.

We have compared the results for which the initial flux rope speed is varied. Figure 11 shows the reconnection electric field 

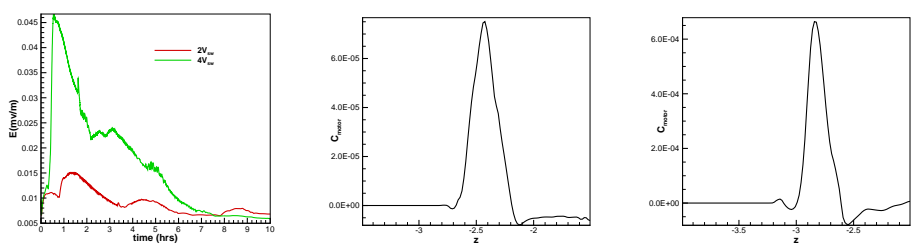

Fig. 11. Model results of a MC moving with different speed. Left: reconnection electric field $E\left(\mathrm{mv} \mathrm{m}^{-1}\right)$ as a function of time with $v_{\max }=2 v_{\mathrm{sw}}$ (red line) and $v_{\max }=4 v_{\mathrm{sw}}$ (green line), middle: profile of $C_{\text {motor }}$ along $z$ direction on the plane of $\phi=0^{\circ}$ for $v_{\max }=2 v_{\mathrm{sw}}$, right: $C_{\text {motor }}$ for $v_{\max }=4 v_{\mathrm{sw}}$.
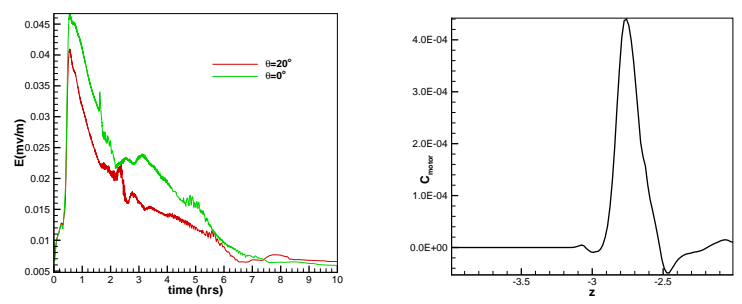

Fig. 12. Model results of a MC moving with different speed direction. The angle between the speed direction of MC and $z$ axis is marked as $\theta$. Left: reconnection electric field $E\left(\mathrm{mv} \mathrm{m}^{-1}\right)$ as a function of time with $\theta=20^{\circ}$ (red line) and $\theta=0^{\circ}$ (green line), right: profile of $C_{\text {motor }}$ along $z$ direction on the plane of $\phi=0^{\circ}$ for $\theta=20^{\circ}$.

$E\left(\mathrm{mv} \mathrm{m}^{-1}\right)$ as a function of time for a MC moving with different speed $v_{\max }$ and the profile of $C_{\text {motor }}$ along $z$ direction on the plane of $\phi=0^{\circ} . C_{\text {motor }}=\boldsymbol{V} \cdot(\boldsymbol{J} \times \boldsymbol{B})$ (Ugai 2001; Zhang et al. $2011)$ is a parameter which can be used to measure the efficiency of the magnetic energy converted into the kinetic and thermal energy of plasma. The $C_{\text {motor }}$ in this figure is along the $z$ direction through the reconnection sites with $x=32$ for $v_{\max }=2 v_{\mathrm{sw}}$, and $x=30$ for $v_{\max }=4 v_{\mathrm{sw}}$. For the middle figure, the time is at $t=1.5 \mathrm{~h}$, and for the right figure it is $t=1 \mathrm{~h}$, which corresponds to the time at which the reconnection electric fields are the largest. Figure 11 shows that $E$ rises quickly soon after the beginning of simulation, followed by a gradual decaying phase as the flux-ropes shrink. The global maximum reconnection rate for $v_{\max }=4 v_{\mathrm{sw}}$ is $0.045 \mathrm{mv} \mathrm{m}^{-1}$ at $t=1 \mathrm{~h}$ (first peak), for $v_{\max }=2 v_{\mathrm{sw}}$ is $0.015 \mathrm{mv} \mathrm{m}^{-1}$ at $t=1.5 \mathrm{~h}$. This is approximately the same after $t=7 \mathrm{~h}$ for the two cases. The beginning time of reconnection for $v_{\max }=4 v_{\mathrm{sw}}$ is at $t=0.5 \mathrm{~h}$, for $v_{\max }=2 v_{\mathrm{sw}}$ is at $t=1 \mathrm{~h}$. So the relative motion of the MC and HCS influence the reconnection rate and reconnection time, which can also be seen from the middle and right figures. The $C_{\text {motor }}$ for $v_{\max }=4 v_{\mathrm{sw}}$ is far bigger than $v_{\max }=2 v_{\mathrm{sw}}$, that means the efficiency of the magnetic energy converted into the kinetic and thermal energy of plasma is large and the reconnection rate for $v_{\max }=4 v_{\mathrm{sw}}$ is bigger. Figure 12 shows the reconnection electric field $E\left(\mathrm{mv} \mathrm{m}^{-1}\right)$ as a function of time for a $\mathrm{MC}$ moving with different speed direction $\theta$ and the profile of $C_{\text {motor }}$ along $z$ direction on the plane of $\phi=0^{\circ}$. The $C_{\text {motor }}$ in this figure is along the $z$ direction through the reconnection site with $x=32$ for $\theta=20^{\circ}$ at $t=1 \mathrm{~h}$. The reconnection time when the reconnection electric field are the largest are the same for $\theta=20^{\circ}$ and $\theta=0^{\circ}$. The only difference is the reconnection site and the maximum reconnection rate. The global maximum reconnection rate for $\theta=0^{\circ}$ is $0.045 \mathrm{mv} \mathrm{m}^{-1}$, for $\theta=20^{\circ}$ is $0.04 \mathrm{mv} \mathrm{m}^{-1}$ at $t=1 \mathrm{~h}$. The $C_{\text {motor }}$ for $\theta=0^{\circ}$ is bigger than $\theta=20^{\circ}$. So the reconnection rate is dependent on the magnitude and direction of velocity in the MC.

\section{Conclusions and discussions}

We used a 3D MHD numerical model to simulate the reconnection process between MC and HCS in this paper. The 3D MHD numerical model adopt HLL scheme within a FV framework by splitting the MHD equations into two parts: fluid and magnetic induction (Feng et al. 2014). Since the HLL scheme only captures the two fastest waves accurately, and its resolution for the other waves is therefore insufficient, in this paper we used the HLL numerical flux in the fluid part plus some antidiffusion terms to improve the overall efficiency of the scheme (Dedner et al. 2005; Wesenberg 2010).

To initiate the MC, we adopted a very simple spherical plasmoid model which is superposed on the background solar wind, and the model contains a high speed, high density and high pressure spherical plasmoid (Zhou et al. 2012; Zhou \& Feng 2013). The direction of the magnetic field in MC is anticlockwise. We first roughly estimated the numerical diffusion computed in our code. We compared the maximum current density with time when a MC moves to HCS using different magnetic diffusivity coefficients, the results suggest that effective numerical diffusion is on the order of $5 \times 10^{-5}$ in our code, which is acceptable.

When the MC moving toward the current sheet, the plasmoid rapidly expands since the injected MC has higher plasma density, speed, and magnetic pressure. As the MC moves toward the current sheet, the current sheet becomes thinner and reconnection taked place as the MC and solar wind have antiparallel magnetic lines. The reconnection takes place mainly at the leading edge of the flux rope, which is a driven reconnection. The HCS is deformed and bent during reconnection, and we also find reconnection take place at rear edge of the flux rope. An observer passes through the numerical test region of the magnetic reconnection will find the basic features of magnetic reconnections (Wei et al. 1997, 2005). We also identify the reconnection exhaust region, using anticorrelated changes in $\boldsymbol{B}$ and $\boldsymbol{V}$ at the leading edge of the field reversal region and correlated variations in $\boldsymbol{B}$ and $\boldsymbol{V}$ at the trailing edge (Gosling et al. 2007; Gosling 2008; Gosling \& Phan 2013; Wang et al. 2010c). The exhaust was associated with a small increase in proton temperature and a decrease in magnetic field strength, there has a decrease in the proton number densities within the exhaust. From the exhaust-oriented coordinate system $(L, M, N)$ derived from minimum variance analysis of the filtered magnetic field data, the dimensionless reconnection rate was calculated to be 0.07 .

We changed the magnetic field direction in $\mathrm{MC}$ and the direction of the magnetic field in MC become clockwise. The positions at which the reconnection takes place is different to the above case, in that the magnetic field in MC is anticlockwise. The reconnection appears to be primarily located at the leading edge of the flux rope soon after the beginning of simulation. As the MC moves to the HCS, the reconnection at the leading edge of the flux rope terminate, and we find reconnection take place at two sides of the flux rope, which can be interpreted as the fluid behind the MC squeeze the MC and arouse the driven reconnection Wei et al. (2002). Also, the reconnection rate is larger in this case. Then, we want to see the effect of magnetic fields magnitude of $\mathrm{MC}$ on the reconnection rate. We ran three cases with the magnetic fields magnitude are $600 \mathrm{nT}$, $500 \mathrm{nT}$, and $300 \mathrm{nT}$, respectively. We find that the reconnection rate becomes larger by increasing the magnetic field magnitude in MC. Finally, we also compared the results where the initial flux rope speed are varied, such as the magnitude and direction of velocity in the $\mathrm{MC}$. We find that the reconnection rate becomes larger by increasing the relative motion of the MC and 
HCS, and decreasing the angle between the speed direction of $\mathrm{MC}$ and $z$ axis also make the reconnection rate become larger. The large reconnection rate correspond to the large $C_{\text {motor }}$ which means the efficiency of the magnetic energy converted into the kinetic and thermal energy of plasma. It needs to be noted that the magnitude of the velocity also affects the beginning time of reconnection.

In this paper we only attempt to describe the reconnection process between MC and HCS by means of the numerical study, and the computational domain here in $r$ direction only reaches $110 R_{\mathrm{s}}$. To understand the magnetic reconnection phenomena under interplanetary conditions, it should be necessary to use the numerical simulation with high accurate and the low viscosity algorithm. Further work should put emphasis on the reconnection process in the solar-terrestrial space with a highorder numerical scheme, then to find the major ingredients which affect the MC's final arrival time and strength at Earth.

Acknowledgements. The work is jointly supported by the National Natural Science Foundation of China (Grant Nos. 41504132, 41731067, 41531073, and 41574171) and the Specialized Research Fund for State Key Laboratories. The work was carried out at National Supercomputer Center in Tianjin, China and the calculations were performed on TianHe-1 (A). We sincerely thank the anonymous referee for helpful suggestions.

\section{References}

Alexandrova, O., Chen, C. H. K., Sorriso-Valvo, L., Horbury, T. S., \& Bale, S. D. 2013, Space Sci. Rev., 178, 101

Birn, J., \& Hesse, M. 2001, J. Geophys. Res. Atmos., 106, 3737

Borovsky, J. E., Hesse, M., Birn, J., \& Kuznetsova, M. M. 2008, J. Geophys Res. Atmos., 113, A07210

Braginskii, S. I. 1965, Rev. Plasma Phys., 1, 205

Cranmer, S. R., Matthaeus, W. H., Breech, B. A., \& Kasper, J. C. 2009, ApJ, 702,1604

Cranmer, S. R., Gibson, S. E., \& Riley, P. 2017, Space Sci. Rev., 212, 1

Dedner, A., Kröner, D., Rohde, C., \& Wesenberg, M. 2005, in Radiation magnetohydrodynamics: analysis for model problems and efficient 3D simulations for the full system (Berlin Heidelberg: Springer)

Endeve, E., Leer, E., \& Holzer, T. E. 2003, ApJ, 589, 1040

Feng, X., Hu, Y., \& Wei, F. 2006, Sol. Phys., 235, 235

Feng, X., Zhang, M., \& Zhou, Y. 2014, ApJS, 214, 6

Gibson, S. E., \& Fan, Y. 2008, J. Geophys. Res. Space Phys., 113, A09103

Gombosi, T. I., van der Holst, B., Manchester, W. B., \& Sokolov, I. V. 2018, Liv. Rev. Sol. Phys., 15, 4

Gosling, J. T. 2008, Proc. IAU, 4, 367

Gosling, J. T. 2012, Space Sci. Rev., 172, 187

Gosling, J. T., \& Phan, T. D. 2013, ApJ, 763, L188

Gosling, J. T., Mccomas, D. J., Phillips, J. L., et al. 1994, Geophys. Res. Lett., 21,2271

Gosling, J. T., Skoug, R. M., Mccomas, D. J., \& Smith, C. W. 2005, J. Geophys. Res. Atmos., 110, 377

Gosling, J. T., Eriksson, S., \& Schwenn, R. 2006a, J. Geophys. Res. Space Phys. 111
Gosling, J. T., Mccomas, D. J., Skoug, R. M., \& Smith, C. W. 2006b, Geophys. Res. Lett., 33, 254

Gosling, J. T., Eriksson, S., Blush, L. M., et al. 2007, Geophys. Res. Lett., 34 Hartle, R. E., \& Sturrock, P. A. 1968, ApJ, 151, 95

Hollweg, J. V. 1976, J. Geophys. Res., 79, 3845

Hollweg, J. V., \& Isenberg, P. A. 2002, J. Geophys. Res. Space Phys., 107, 12

Holzer, T. E., \& Axford, W. I. 1970, ARA\&A, 8, 163

Holzer, T. E., Leer, E., \& Zhao, X. P. 1986, J. Geophys. Res. Space Phys., 91, 4126

Jin, M., Manchester, W. B., van der Holst, B., et al. 2017a, ApJ, 834, 172

Jin, M., Manchester, W. B., van der Holst, B., et al. 2017b, ApJ, 834, 173

Kataoka, R., Ebisuzaki, T., Kusano, K., et al. 2009, J. Geophys. Res. Atmos., 114

Lavraud, B., Gosling, J. T., Rouillard, A. P., et al. 2009, Sol. Phys., 256, 379

Lavraud, B., Owens, M. J., \& Rouillard, A. P. 2011, Sol. Phys., 270, 285

Lavraud, B., Ruffenach, A., Rouillard, A. P., et al. 2014, J. Geophys. Res. Space Phys., 119, 26

Li, B., Habbal, S. R., Li, X., \& Mountford, C. 2005, J. Geophys. Res. Space Phys., 110, A12

Lionello, R., Linker, J. A., \& Mikić, Z. 2001, ApJ, 546, 542

Marsch, E. 1999, Space Sci. Rev., 87, 1

Marsch, E. 2006, Liv. Rev. Sol. Phys., 3, 1

Odstrcil, D., \& Karlicky, M. 1997, A\&A, 326, 1252

Ofman, L. 2004, J. Geophys. Res. Space Phys., 109, A7

Parker, E. N., Marshak, R. E., \& Johnson, Jr., G. 1964, Phys. Today, 17, 72

Plunkett, S. P., \& Wu, S. T. 2000, IEEE Trans. Plasma Sci., 28, 1807

Riley, P., Ko, Y. K., Hansteen, V. H., et al. 2016, Space Sci. Rev., 201, 1

Ruffenach, A., Lavraud, B., Owens, M. J., et al. 2012, J. Geophys. Res. Space Phys., 117, 1996

Ruffenach, A., Lavraud, B., Farrugia, C. J., et al. 2014, J. Geophys. Res. Space Phys., 120, 43

Schmidt, J. M. 2000, Sol. Phys., 197, 135

Schmidt, J. M., \& Cargill, P. J. 2001, J. Geophys. Res. Atmos., 106, 8283

Schmidt, J. M. \& Cargill, P. J. 2003, J. Geophys. Res. Atmos., 108, SSH51

Schwartz, S. J., Zweibel, E. G., \& Goldman, M. 2013, Space Sci. Rev., 178, 81

Shiota, D., Kusano, K., Miyoshi, T., \& Shibata, K. 2010, ApJ, 718, 1305

Sturrock, P. A., \& Hartle, R. E. 1966, Phys. Rev. Lett., 16, 628

Taubenschuss, U., Erkaev, N. V., Biernat, H. K., et al. 2010, Ann. Geophys., 28, 1075

Totten, T. L., Freeman, J. W., \& Arya, S. 1995, J. Geophys. Res. Space Phys., 100,13

Ugai, M. 2001, Space Sci. Rev., 95, 601

Wang, R., Lu, Q., Du, A., \& Wang, S. 2010a, Phys. Rev. Lett., 104, 175003

Wang, R., Lu, Q., Li, X., Huang, C., \& Wang, S. 2010b, J. Geophys. Res. Atmos., 115,11201

Wang, Y., Wei, F. S., Feng, X. S., et al. 2010c, Phys. Rev. Lett., 105, 195007

Wang, L., Hakim, A. H., Bhattacharjee, A., \& Germaschewski, K. 2015, Phys. Plasmas, 22, 603

Wang, L., Germaschewski, K., Hakim, A., et al. 2018, J. Geophys. Res. Space Phys., 123, 2815

Wei, F., Schwenn, R., \& Hu, Q. 1997, Sci. Chin. Technol. Sci., 40, 463

Wei, F., Liu, R., Fan, Q., \& Feng, X. 2002, Sci. Chin. Technol. Sci., 32, 608

Wei, F., Feng, X., \& Hu, Q. 2005, Adv. Space Res., 36, 2303

Wesenberg, M. 2010, J. Numer. Math., 10, 37

Zhang, S. H., Feng, X. S., Wang, Y., \& Yang, L. P. 2011, Chin. Phys. Lett., 28, 089601

Zhou, Y. F., \& Feng, X. S. 2013, J. Geophys. Res. Space Phys., 118, 6007

Zhou, Y. F., Feng, X. S., Wu, S. T., et al. 2012, J. Geophys. Res., 117, A01102

Ziegler, U. 2004, J. Comput. Phys., 196, 393

Ziegler, U. 2011, J. Comput. Phys., 230, 1035 